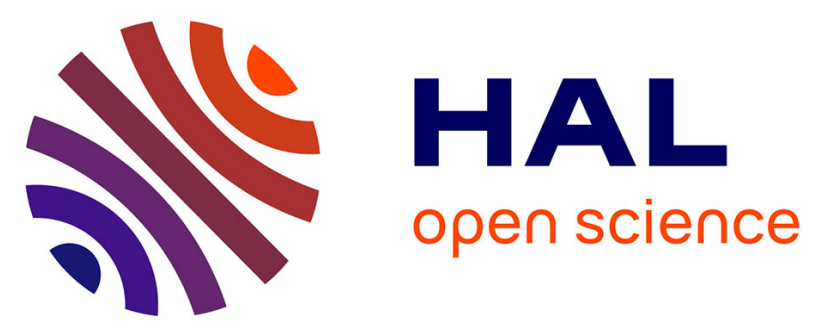

\title{
A Comparative Study of Characteristic Finite Element and Characteristic Finite Volume Methods for Convection-Diffusion-Reaction Problems on Triangular Grids
}

\author{
Sutthisak Phongthanapanich, Robert Eymard
}

\section{To cite this version:}

Sutthisak Phongthanapanich, Robert Eymard. A Comparative Study of Characteristic Finite Element and Characteristic Finite Volume Methods for Convection-Diffusion-Reaction Problems on Triangular Grids. Applied Science and Engineering Progress, 2019, 12 (4), pp.1-8. 10.14416/j.asep.2019.10.002 . hal-03180538

\section{HAL Id: hal-03180538 \\ https://hal.science/hal-03180538}

Submitted on 29 Mar 2021

HAL is a multi-disciplinary open access archive for the deposit and dissemination of scientific research documents, whether they are published or not. The documents may come from teaching and research institutions in France or abroad, or from public or private research centers.
L'archive ouverte pluridisciplinaire HAL, est destinée au dépôt et à la diffusion de documents scientifiques de niveau recherche, publiés ou non, émanant des établissements d'enseignement et de recherche français ou étrangers, des laboratoires publics ou privés. 


\section{A Comparative Study of Characteristic Finite Element and Characteristic Finite Volume Methods for Convection-Diffusion-Reaction Problems on Triangular Grids}

Sutthisak Phongthanapanich

Department of Mechanical Engineering Technology, College of Industrial Technology,

King Mongkut's University of Technology North Bangkok, Bangkok, Thailand

Robert Eymard

Université Paris-Est Marne-la-Vallée 5 boulevard Descartes Champs-sur-Marne

F-77454 MARNE LA VALLÉE CEDEX 2, France

\begin{abstract}
The paper aims to compare the accuracy and robustness of the characteristic finite element method (CFEM) and characteristic finite volume method (CFVM) for solving convection-diffusionreaction problems on two-dimensional triangular grids. The tests are performed on a square unit domain, to which an advective field is imposed in a domain. The results show that the CFEM gives less accurate solution than CFVM for the rotation of a slotted-cylinder and rotation of Gaussian cone problems. Moreover, CFEM gives oscillate solution while the CFVM provides an oscillationfree solution for the skew flow to the mesh problem.
\end{abstract}

Keywords: Characteristic Finite Element Method, Characteristic Finite Volume Method, ConvectionDiffusion-Reaction, Triangular Grids

\section{Introduction}

Many science and engineering applications require the simulation of time-dependent convection-diffusionreaction equations. The convection is defined as the movement of substance due to the fluid transport medium, the diffusion is referred to the dispersion of a substance involved in the process throughout the physical domain of the problem, and the reaction is the process of interaction through which the substances are generated or consumed. Generally, the scale of the diffusion is smaller by several orders of magnitude compared to the size of the advective flow field. A main feature of the convection- and reaction-dominated is the presence of the sharp layers as a part of the solution. The accurate simulation of such processes requires numerical methods such that able to compute sharp layers without an occurrence of spurious oscillations.

The standard Galerkin finite element method usually yields oscillatory solutions for convection-dominated problems [1]. Many methods have been proposed to overcome the instability and inaccuracy of the solution. The shock-capturing procedure [2] is the usual remedy for these difficulties. Among the stabilizing schemes, the characteristic Galerkin scheme has been developed by applying the time-stepping technique as the basis [3,4]. The explicit characteristic Galerkin scheme based on the Taylor series expansion is an attractive one due to its simple implementation and fast calculation. The scheme uses a characteristic approximation to trace advection in time and combine with a low-order mixed finite element spatial approximation of the equation. Moreover, the scheme is locally conservative on the discrete level such that fluid is transported along with the approximate characteristics.

Recently, the author proposed an explicit characteristic finite volume method that can provide stabilized numerical solutions for pure advection and advectiondominated diffusion problems [5-7]. The idea of the characteristic-based scheme is used to discrete the Lagrangian derivative as the function of time. An explicit finite volume method based on the cell-centered scheme is employed to derive the discretized equations. The Galerkin finite element technique is applied to estimate the gradient quantities at the cell faces. The finite volume method is applied to discretize the equation because of it some of the important features. Firstly, it may be used on arbitrary geometries, using structured or unstructured grids. Secondly, it is the local conservativity of the numerical fluxes, that is the numerical flux is conserved from one discretization cell to its neighbor [8].

The objective of this research work is to compare the computational efficiency of the CFEM and CFVM for solving two-dimensional convection-diffusion-reaction problems on structured triangular grids. The paper is organized as follows. In Section 2, the governing equations and numerical formulations are presented. Then, the schemes are examined by three benchmark test cases to evaluate their accuracy and robustness in Section 3.

\section{The Numerical Scheme}

\subsection{The characteristic convection-diffusion- reaction equation}

The governing differential equation for the twodimensional convection-diffusion-reaction equation is

$$
\frac{\partial \phi}{\partial t}+\nabla \cdot(\mathbf{v} \phi-\varepsilon \nabla \phi)+\kappa \phi=0 \quad \text { in } \quad \Omega \times T
$$

subject to the boundary conditions

$$
\begin{aligned}
& \phi=g_{D} \quad \text { in } \quad \partial \Omega_{D} \\
& \varepsilon \frac{\partial \phi}{\partial \mathbf{n}}=g_{N} \text { in } \partial \Omega_{N}
\end{aligned}
$$

with $\partial \Omega=\partial \Omega_{D} \cup \partial \Omega_{N}$ with $\partial \Omega_{D} \cap \partial \Omega_{N}=\varnothing$, and the initial condition is defined for $x \in \Omega$ with $\Omega \subset R^{2}$ by 


$$
\phi(\mathbf{x}, 0)=\phi_{0}(\mathbf{x})
$$

where $\phi$ is the scalar quantity, $\mathbf{v}=\mathbf{v}(\mathbf{x})$ is the given convection velocity vector, $\varepsilon \geq 0$ is the diffusion coefficient, $\kappa$ is the reaction coefficient, and ${ }^{t \in(0, T]}$ for $T<\infty$.

By applying a characteristic approximation to trace advection in time, as explained in Ref. [5-7] to Eq. (1) yields

$$
\frac{\partial \phi}{\partial t}-\nabla \cdot(\varepsilon \nabla \phi)+\kappa \phi=0
$$

where all terms are to be evaluated at $\mathbf{x}^{\prime}=\mathbf{x}^{\prime}(t)$.

Then, by carrying out a Taylor series expansion, the convection term reappears in the equation along with an additional second-order term. This second-order term acts as a smoothing operator that reduces the oscillations arising from the spatial discretization of the convection terms. The fully explicit characteristic convectiondiffusion-reaction equation is given by

$$
\begin{aligned}
\phi^{n+1}-\phi^{n} & =-\Delta t\left[\mathbf{v} \cdot \nabla \phi^{n}-\epsilon \nabla \cdot \nabla \phi^{n}+\kappa \phi^{n}\right] \\
& +\frac{(\Delta t)^{2}}{2} \mathbf{v} \cdot \nabla\left[\mathbf{v} \cdot \nabla \phi^{n}+\kappa \phi^{n}\right]
\end{aligned}
$$

Together with the use of the divergence-free assumption $\nabla \cdot \mathbf{v}=0$, Equation (6) could be written finally in the conservation form as

$$
\begin{aligned}
\phi^{n+1}-\phi^{n}= & -\Delta t\left[\nabla \cdot\left(\mathbf{v} \phi^{n}-\varepsilon \nabla \phi^{n}\right)+\kappa \phi^{n}\right] \\
& +\frac{(\Delta t)^{2}}{2} \nabla \cdot\left[\mathbf{v}\left(\mathbf{v} \cdot \nabla \phi^{n}+\kappa \phi^{n}\right)\right]
\end{aligned}
$$

\subsection{The characteristic finite element method}

Assuming a variation of $\phi$ within an element can be approximated by

$$
\begin{aligned}
& \phi^{n}=\mathbf{N} \phi_{e}^{n} \\
& \phi^{n+1}=\mathbf{N} \phi_{e}^{n+1}
\end{aligned}
$$

where $\mathrm{N}$ is an element interpolation function. Applying the standard Galerkin weighting technique to Eq. (6) to yield

$$
\begin{aligned}
\int_{\Omega^{\prime}} \mathbf{N}\left(\frac{\phi^{n+1}-\phi^{n}}{\Delta t}\right) d A & =-\int_{\Omega^{n}} \mathbf{N}\left(\mathbf{v} \cdot \nabla \phi^{n}\right) d A \\
& +\int_{\Omega^{\prime}} \mathbf{N}\left(\frac{\Delta t}{2} \mathbf{v} \cdot \nabla\left(\mathbf{v} \cdot \nabla \phi^{n}\right)\right) d A \\
& +\int_{\Omega^{n}} \mathbf{N}\left(\nabla \cdot\left(\varepsilon \nabla \phi^{n}\right)\right) d A \\
& -\kappa \int_{\Omega} \mathbf{N}\left(\phi^{n}-\frac{\Delta t}{2} \mathbf{v} \cdot \nabla \phi^{n}\right) d A
\end{aligned}
$$

Because the hyperbolic nature of the convectiondominated equation, a nonlinear viscous shock capturing operator is added to minimize the oscillations at the discontinuities in the curvature of the front. The operator [9] is given by

$$
B_{s c}(\phi)=v_{s c}(\phi) \int_{\Omega^{\prime}}\left(\nabla \mathbf{N} \cdot \nabla \mathrm{N}^{\top}\right) \phi^{n} d A
$$

and

$v_{s c}(\phi)=\sigma_{x} h^{e}\left|\frac{\partial \phi}{\partial t}+\mathbf{v} \cdot \nabla \phi^{\prime \prime}\right| /\left(\left|\mathbf{v} \cdot \nabla \phi^{n}\right|+h^{e}\right)$

where $\sigma_{s c}$ is a parameter used to control added viscosity (typically is 0.1 ), and $h^{e}$ is a measure of the element $e$. Finally, applying the Green-Gauss theorem to Eq. (9) also, using Eq. (8) to obtain

$$
\begin{aligned}
\frac{\mathbf{M}_{\phi}}{\Delta t}\left(\phi_{e}^{n+1}-\phi_{e}^{n}\right)= & -\mathbf{C}_{1}^{\phi}-\mathbf{C}_{2}^{\phi}-\mathbf{C}_{3}^{\phi}-\mathbf{K}_{1}^{\phi}+\mathbf{K}_{2}^{\phi}+\mathbf{K}_{x} \\
& +\mathbf{f}_{K 1}^{\phi}+\mathbf{f}_{C 2}^{\phi}
\end{aligned}
$$

where

$$
\begin{aligned}
& \mathbf{M}_{\phi}=\int_{\Omega^{\top}} \mathrm{NN}^{\mathrm{T}} d A \\
& \mathbf{C}_{1}^{\varphi}=\int_{\Omega^{e}} \mathbf{N}\left(\mathbf{v} \cdot \nabla \mathbf{N}^{T}\right) d A \phi_{e}^{n} \\
& \mathbf{C}_{2}^{\phi}=\varepsilon \int_{\Omega^{\natural}} \nabla \mathbf{N} \cdot \nabla \mathbf{N}^{T} d A \phi_{e}^{n} \\
& \mathbf{C}_{3}^{\varphi}=\kappa \int_{\Omega} \mathbf{N N}^{T} d A \phi_{e}^{n} \\
& \mathbf{K}_{1}^{\phi}=\frac{\Delta t}{2} \int_{\Omega^{*}}(\mathbf{v} \cdot \nabla \mathbf{N})\left(\mathbf{v} \cdot \nabla \mathbf{N}^{T}\right) d A \phi_{e}^{n} \\
& \mathbf{K}_{2}=\kappa \frac{\Delta t}{2} \int \mathbf{N}\left(\mathbf{v} \cdot \nabla \mathbf{N}^{T}\right) d A \phi_{e}^{n} \\
& \mathbf{K}_{s c}^{\phi}=v_{s}(\phi) \int_{\Omega} \nabla \mathbf{N} \cdot \nabla \mathbf{N}^{\top} d A \phi_{e}^{n} \\
& \mathbf{f}_{K 1}^{\phi}=\frac{\Delta t}{2} \int_{\Gamma} \mathbf{N}(\mathbf{v} \cdot \hat{\mathbf{n}})\left(\mathbf{v} \cdot \nabla \mathbf{N}^{T}\right) d S \phi_{l}^{n} \\
& \mathbf{f}_{C 2}=\varepsilon \int_{\Gamma^{\circ}} \mathbf{N}\left(\hat{\mathbf{n}} \cdot \nabla \mathbf{N}^{T}\right) d S \phi_{i}^{n}
\end{aligned}
$$

\subsection{The characteristic finite volume method}


The computational domain is first discretized into a collection of non-overlapping control volumes $\Omega_{i} \in \Omega, i=1, \ldots, N$, that completely cover the domain such that $\Omega=\cup \Omega_{i=1}, \Omega_{j} \neq 0$ and $\Omega_{i} \cap \Omega_{j}=0$ if $i \neq j$. To obtain the finite volume equation, Eq. (7) is then integrated over the control volume $\Omega_{i}$ to yield

$$
\begin{aligned}
\int_{\Omega^{2}}\left(\phi^{n+1}-\phi^{n}\right) d A= & \int_{\Omega^{n}}\left(-\Delta t\left[\nabla \cdot\left(\mathbf{v} \phi^{n}-\varepsilon \nabla \phi^{n}\right)+\kappa \phi^{n}\right]\right) \\
& \left.+\frac{(\Delta t)^{2}}{2} \nabla \cdot\left[\mathbf{v}\left(\mathbf{v} \cdot \nabla \phi^{n}+\kappa \phi^{n}\right)\right]\right) d A
\end{aligned}
$$

Then the divergence theorem is applied to the spatial terms to yield a fully explicit characteristic-based scheme for solving Eq. (7) in the form

$$
\begin{aligned}
\phi_{i}^{n+1}= & \phi_{i}^{n}-\frac{\Delta t}{\left|\Omega_{i}\right|} \sum_{j=1}^{N_{i}}\left|\Gamma_{i j}\right| \hat{\mathbf{n}}_{i j} \cdot\left[\left(\mathbf{v}_{i j}^{n} \phi_{j,}^{n}-\varepsilon \nabla \phi_{i j}^{n}\right)\right. \\
& \left.-\frac{\Delta t}{2} \mathbf{v}_{i j}^{n}\left(\mathbf{v}_{i}^{n} \cdot \nabla \phi_{i}^{n}\right)\right]
\end{aligned}
$$

where $N_{1}$ is a number of adjacent cell faces, $\Gamma_{i j}$ is the segment of the boundary ${ }^{\partial \Omega_{i}}$ between the two adjacent control volumes $\Omega_{i}$ and $\Omega_{j}$, and the quantities at the time $t=n$ are defined by

$$
\begin{aligned}
& \phi_{i j}^{n}=\phi_{i j}\left(t^{n}\right) \\
& \phi_{i}^{n}=\phi_{i}\left(t^{n}\right)
\end{aligned}
$$

Finally, the scalar quantity at the cell faces, $\phi_{i j}^{n}$, is approximated by applying Taylor's series expansion in space such that

$\phi_{i j}^{n}=\phi_{i}^{n}+\left(\mathbf{x}_{i j}-\mathbf{x}_{i}\right) \cdot \nabla \phi_{i}^{n}$

For the opposite direction of velocity, the values $\phi_{i j}^{n}$ could be computed from Eq. (27), but using the values from the neighboring cells according to the upwinding direction.

Moreover, in this paper, the gradient term, $\nabla \phi_{i j}^{n}$, is approximated by the weighted residuals method, which is commonly used in the finite element technique [6].

Finally, to ensure the stability of a numerical scheme, the CFL-like stability criterion must be fulfilled. In this paper, the time-step within each control volume $i$ for both the CFEM and CFVM is determined from

$$
\Delta t=C \min _{i}\left(\frac{\left|\Omega_{i}\right|}{\max _{j=1, N_{j}}\left|\mathbf{v}_{n, i, j}\right|}, \frac{\left|\Gamma_{i j}^{c}\right|^{2}}{2 \varepsilon}\right)
$$

where $\mathbf{v}_{n, i j}$ is the scaled normal velocity at $\Gamma_{i j},\left|\Gamma_{i j}^{c}\right|$ is the cell characteristic length, and $0<C \leq 1$.

\section{Test Problems and Discussion}

In order to illustrate the accuracy and robustness of these numerical schemes, three examples are examined by implementing a second-order accurate scheme. These examples are (1) the rotation of a slotted-cylinder, (2) the rotation of a Gaussian cone, and (3) the skew flow to the mesh. All examples presented in this section were tested on structured triangular grids.

\subsection{Rotation of a slotted-cylinder}

This first problem is adapted from the rotation of a slotted cylinder problem, which was introduced by Zalesak [10]. It is a challenging pure-convection problem because the sudden change of the initial conditions and the shape of the rectangular slot in the domain $\Omega=[0,1]^{2}$ are difficult to capture by most numerical schemes. The rotating velocity field is imposed such that $\mathbf{v}(\mathbf{x})=(y-0.5,0.5-x)$. The initial condition $\phi(\mathbf{x}, 0)$ is given by

$$
\phi(\mathbf{x}, 0)=\left\{\begin{array}{cc}
1 & (r \leq 0.15) \cap(|x-0.15| \\
0 & \geq 0.025 \cup y \geq 0.8) \\
0 & \text { otherwise }
\end{array}\right.
$$

where $r$ is the distance from the center of the domain $(0.5,0.5)$. The time step for this example is set to be equal to the period required for one turn rotation. This example is performed on a uniform grid of $256 \times 256$. The exact solution at the final time step is then equal to the initial condition that is depicted in Figs. (1) and (2).

Figures 3 and 4 show that the CFEM scheme gives a dissipative solution and inaccurate position of a slottedcylinder. Moreover, Figs. 5 and 6 show that the CFVM scheme gives more sharpen and accurate solution.

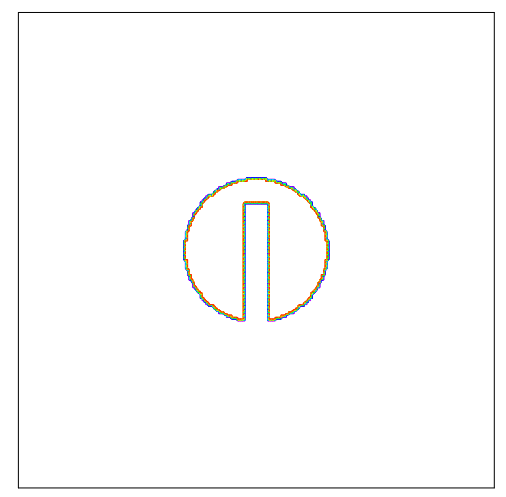

Figure 1: Exact solution at the final time of problem 3.1: 2D plot. 


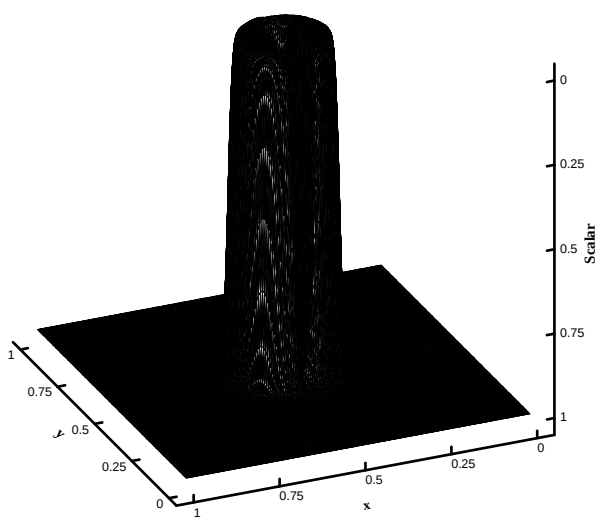

Figure 2: Exact solution at the final time of problem 3.1: 3D plot.

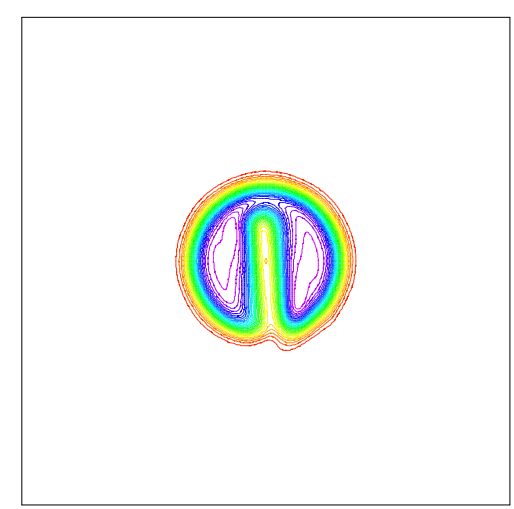

Figure 3: CFEM solution at the final time of problem 3.1: $2 \mathrm{D}$ plot.

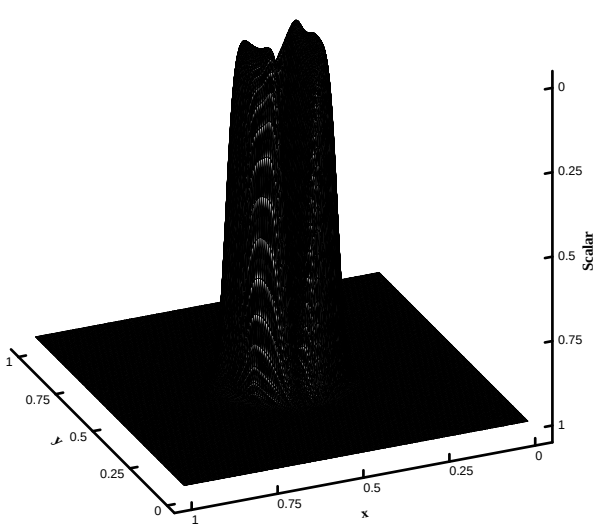

Figure 4: CFEM solution at the final time of problem 3.1: 3D plot.

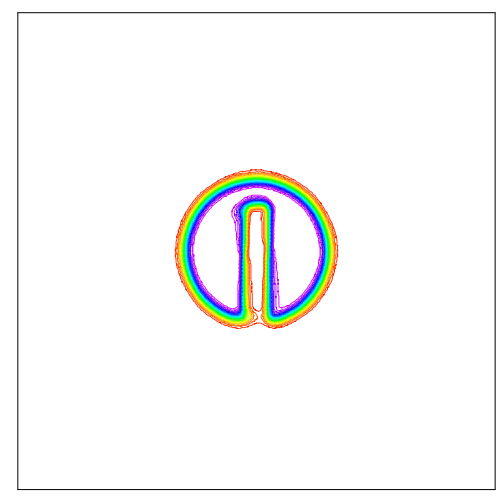

Figure 5: CFVM solution at the final time of problem 3.1: 2D plot.

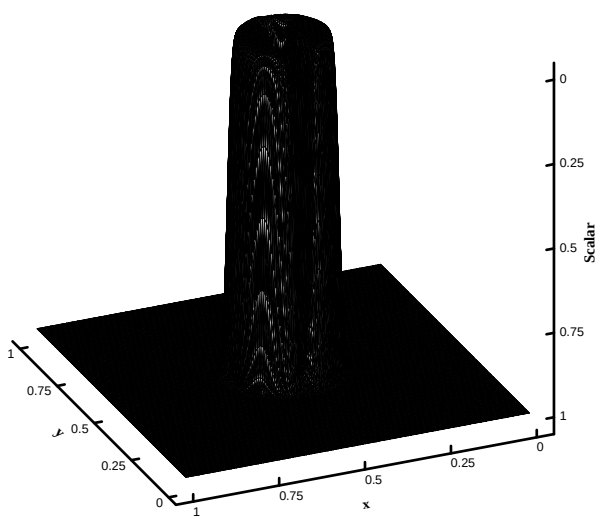

Figure 6: CFVM solution at the final time of problem 3.1: 3D plot.

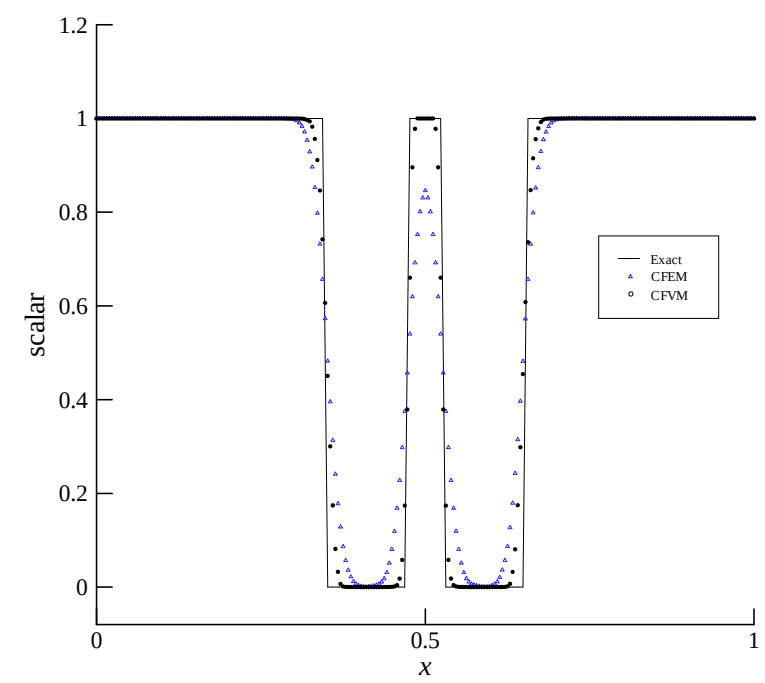

Figure 7: Comparison of the solution along the line $y=0.5$ at the final time of problem 3.1.

Finally, the comparison of the solution along $y=0.5$ is illustrated in Fig. 7. It is seen that the CFVM scheme gives a more accurate solution than the CFEM scheme. It can capture the maximum and minimum values around the slot of the cylinder accurately.

\subsection{Rotation of a Gaussian cone}

The second example is the rotation of a Gaussian pulse in a square domain where the scalar field is rotated around the domain $\Omega=[-0.5,0.5]^{2}$. The rotating velocity field with the angular velocity of $4 \mathrm{rad} / \mathrm{s}$ is imposed as $\mathbf{v}(\mathbf{x})=(-4 y, 4 x)$. This example has been used widely for testing the numerical artifacts that occur on different schemes [11,6]. The initial condition $\phi(\mathbf{x}, 0)$ is given by

$\phi(\mathbf{x}, 0)=\exp \left(-\frac{(x+0.25)^{2}+y^{2}}{2 \sigma^{2}}\right)$ 
where $\sigma=0.0447$. The final time is equal to $\pi / 2$, which is the time period required for one turn rotation. this example is performed on a uniform grid of $128 \times 128$. The diffusion coefficient of $\varepsilon=10^{-4}$, in order to evaluate the robustness and accuracy of the method on a convection-dominated diffusion problem. The 2D and 3D plots of the exact solution as shown in Figs. 8 and 9. Figures 10 and 11 show the numerical solution obtained from the CFEM scheme. Then the numerical solution obtained from the CFVM scheme is also shown in Figs. 12 and 13. Moreover, the comparison of the numerical and exact solutions along the line ${ }^{y=0}$ are presented in Fig. 14.

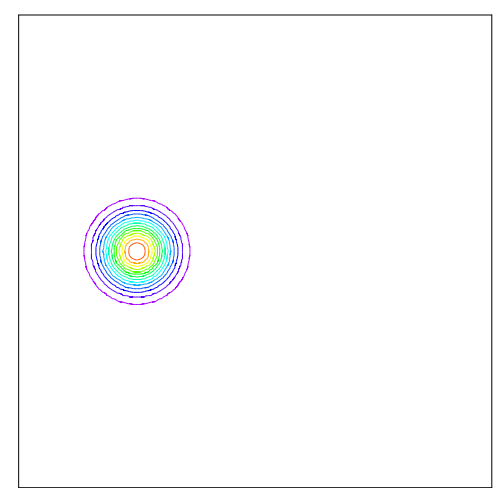

Figure 8: Exact solution at the final time of problem 3.2: $2 \mathrm{D}$ plot.

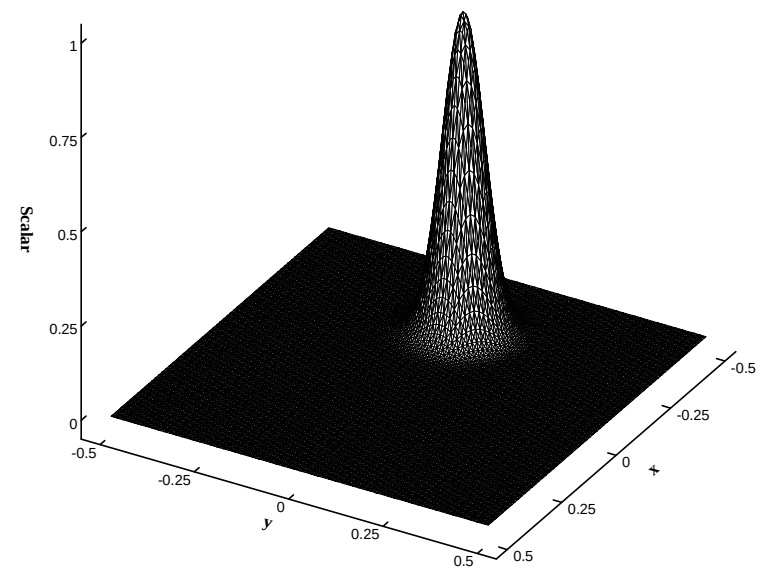

Figure 9: Exact solution at the final time of problem 3.2: 3D plot.

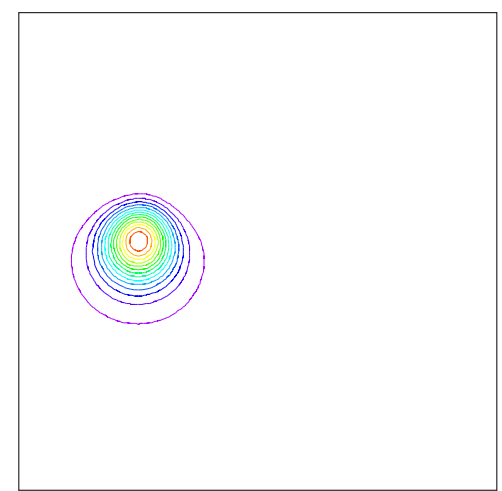

Figure 10: CFEM solution at the final time of problem 3.2: 2D plot.

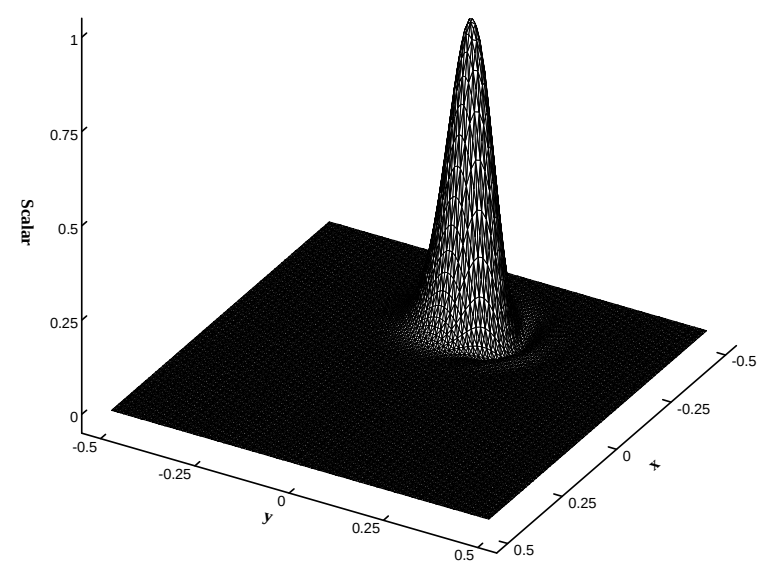

Figure 11: CFEM solution at the final time of problem 3.2: 3D plot.

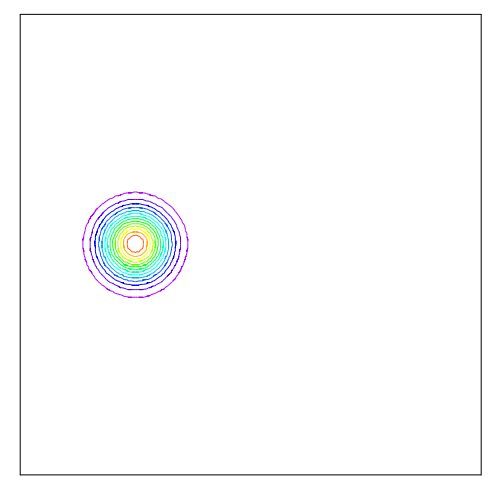

Figure 12: CFVM solution at the final time of problem 3.2: 2D plot.

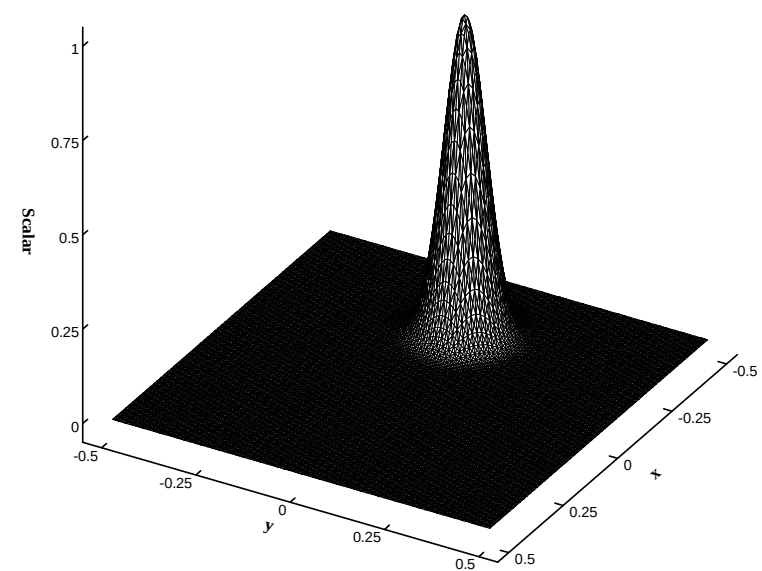

Figure 13: CFVM solution at the final time of problem 3.2: 3D plot. 


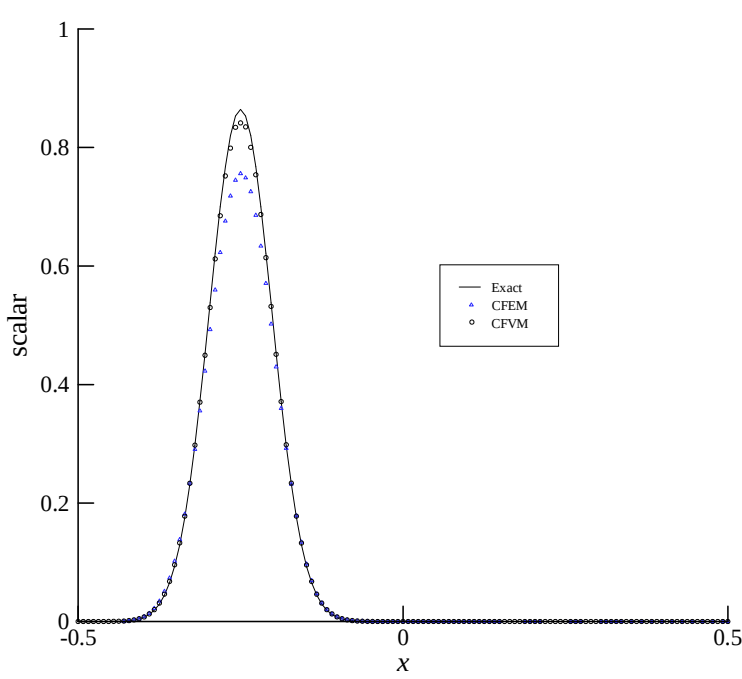

Figure 14: Comparison of the solution along the line $y=0$ at the final time of problem 3.2.

\subsection{Skew flow to the mesh}

The last example is the skew flow problem presented by Hauke [12,7]. This example considers the behavior of the numerical solutions under the convection-reactiondominated diffusion condition in a square domain $\Omega=[0,1]^{2}$, with the initial condition $\phi(\mathbf{x}, 0)=0$. The four boundary conditions are $\phi(x=0)=\phi(y=1)=1$ and $\phi(x=1)=\phi(y=0)=0$. The velocity field is given by $\mathbf{v}(\mathbf{x})=(\cos (\pi / 6), \sin (\pi / 6))$. The numerical solutions are examined in the steady-state condition. It is noted that the influence of boundary conditions on the algorithm is remarkable and leads to instability [12]. The parameters for this test are $\varepsilon=0.005$ and $\kappa=100$. The analyses of these test cases are performed on the $10 \times 10$ grid size.

The 3D plots of numerical solutions of the CFEM and CFVM schemes are depicted in Figs. 15 and 16, respectively. Figure 15 shown that the CFEM scheme provides the oscillate solution along the boundaries $\phi(x=0)=\phi(y=1)=1$ due to the abrupt change of the solutions. Moreover, the CFVM scheme gives nonoscillate solution along these boundaries. The examples confirmed that the CFVM scheme is an accurate and robust scheme for computing a wide range of convectiondominated diffusion-reaction problems.

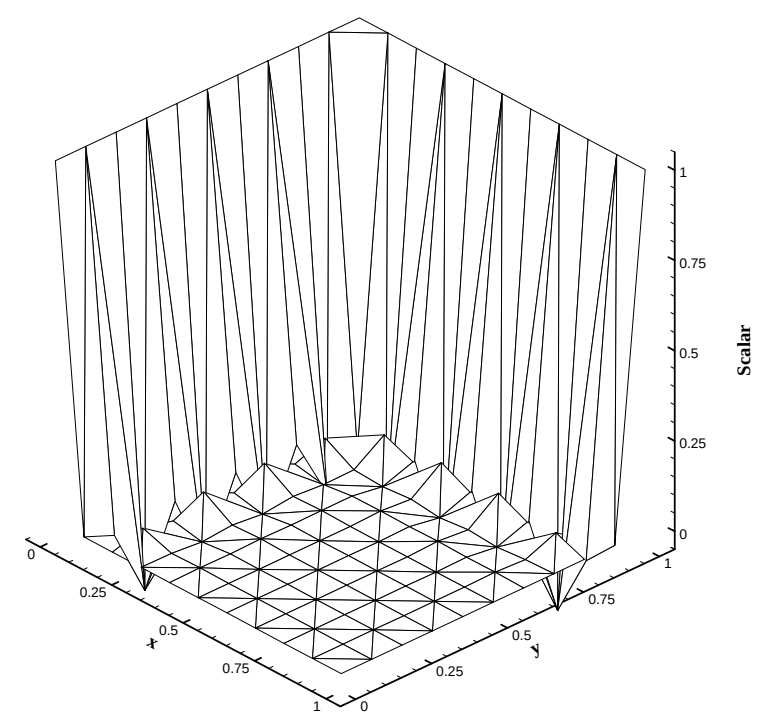

Figure 15: CFEM solution at the final time of problem 3.3: 3D plot.

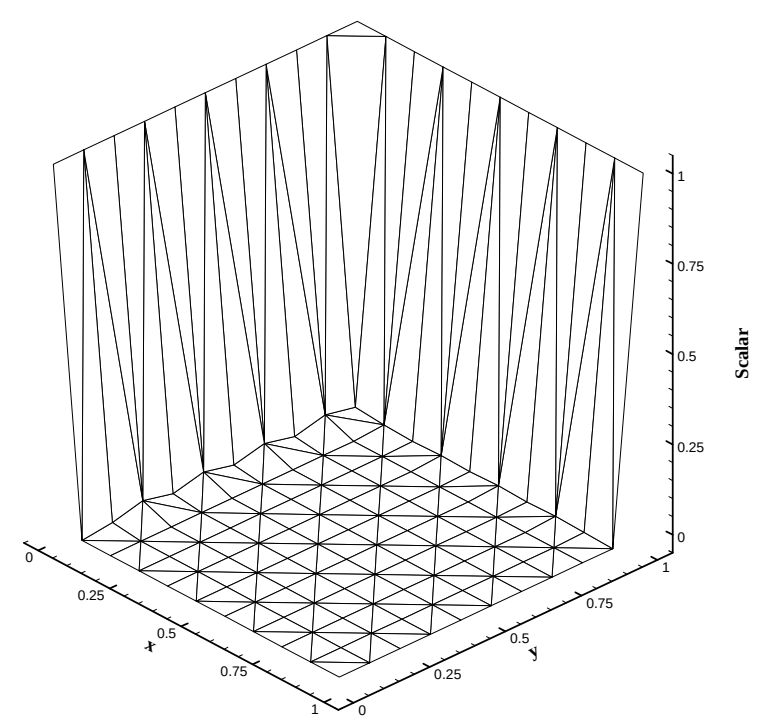

Figure 16: CFVM solution at the final time of problem 3.3: 3D plot.

\section{Concluding Remarks}

The accuracy and robustness of the CFEM and CFVM schemes for solving two-dimensional convectiondiffusion-reaction problems on structured triangular grids are investigated in this paper. The characteristic convection-diffusion-reaction equation is derived. The finite element and finite volume is then used to discretize the characteristic convection-diffusion-reaction equation. Three examples are used to test the accuracy and robustness of both schemes. For the rotation of a slottedcylinder problem, the CFEM scheme provides the diffusive and inaccurate position of the slotted-cylinder solution while the CFVM scheme gives the sharpen solution with an accurate position of the slotted-cylinder solution. The CFEM scheme provides a less accurate solution than the one obtained from the CFVM scheme for rotation of a Gaussian cone problem. Finally, for the skew flow to the mesh, the CFEM scheme provides the oscillate solution along the boundaries due to the abrupt change of the solutions. Moreover, the CFVM scheme 
gives a non-oscillate solution. We can conclude that the CFVM scheme is an accurate and robust method for solving the convection-dominated problems.

\section{Acknowledgments}

The first author is grateful to the College of Industrial Technology, King Mongkut's University of Technology North Bangkok for funding this research work.

\section{References}

[1] A. N. Brooks, and T. J. R. Hughes, "Streamline Upwind Petrov-Galerkin formulation for convection dominated flows with particular emphasis on the incompressible Navier-Stokes equation," Comput. Methods Appl. Mech. Eng., vol. 32, pp. 199-259, 1982.

[2] R. Codina, “A Discontinuity-capturing crosswind dissipation for the finite element solution of the convection-diffusion equation," Comput. Methods Appl. Mech. Eng., vol. 110, pp. 325-342, 1993.

[3] O. C. Zienkiewicz and R. Codina, “A general algorithm for compressible and incompressible flow - part I. the split, characteristic based scheme," Int. J. Numer. Methods Fluids, vol. 20, pp. 869-885, 1995.

[4] R. Codina, "Comparison of some finite element methods for solving the diffusionconvection-reaction equation," Comput. Methods Appl. Mech. Eng., Vol.156, No.1-4, pp. 185-210, 1998.

[5] S. Phongthanapanich and P. Dechaumphai, “A Characteristic-based Finite Volume
Element Method for Convection-DiffusionReaction Equation," T. Can. Soc. Mech. Eng., vol. 32, pp. 549-560, 2008.

[6] S. Phongthanapanich and P. Dechaumphai, "Explicit characteristic finite volume method for convection-diffusion equation on rectangular grids," J. Chin. Inst. Eng., vol. 34, pp. 239-252, 2011.

[7] S. Phongthanapanich and P. Dechaumphai, "An explicit characteristic finite volume element method for non-divergence free convection-diffusion-reaction equation," Int. J. Adv, Eng. Sci. Appl. Math., vol. 4, pp. 179192, 2012.

[8] R. Eymard, T. Gallouet, and R. Herbin, "Finite volume methods," Handbook for Numerical Analysis, Ph. Ciarlet, J. L. Lions eds, North Holland, 2000, pp. 715-1022.

[9] J. Chessa and T. Belytschko, T. "An extended finite element method for twophase fluids," Transaction of ASME, vol. 70, pp. 10-17, 2003.

[10] S. T. Zalesak, "Fully multidimensional fluxcorrected transport algorithms for fluids," $J$. Comput. Phys., vol. 31, pp. 335-362, 1979.

[11] H. Wang and J. Liu, "Development of CFLfree, explicit schemes for multidimensional advection-reaction equations,” SIAM J. Sci. Comput., vol. 23, pp.1418-1438, 2001.

[12] G. Hauke, “A simple subgrid scale stabilized method for the advection-diffusion-reaction equation," Comput. Methods Appl. Mech. Eng., Vol.191, pp. 2925-2947, 2002. 